\title{
Analysis of Keypoint Detection Algorithms for Mass Candidates Selection in Mammography Images
}

\author{
Felipe Victor de Sá Oliveira ${ }^{1}$, Gersica Agripino Alencar ${ }^{1}$, Filipe Rolim Cordeiro ${ }^{2}$ \\ ${ }^{1}$ Programa de Pós-Graduação em Informática Aplicada \\ Universidade Federal Rural de Pernambuco (UFRPE) - Recife - PE - Brasil \\ ${ }^{2}$ Departamento de Computação \\ Universidade Federal Rural de Pernambuco (UFRPE) - Recife - PE - Brasil \\ \{felipe.sa.oliveirati, agersica, filipe.rolim\}@gmail.com
}

\begin{abstract}
Breast cancer has been a growing problem for women around the world. The correct interpretation of mammographic images is important for the diagnosis of breast cancer. However, this is a difficult task even for a specialist. Image processing is used to make the diagnosis less susceptible to errors. Thus, the present work proposes a new method for the search of lesion candidates in mammographic images. To verify the efficiency of the approach, the behavior of the SURF, SIFT, BRISK and ORB algorithms were analyzed, as well as the Selective Search algorithm for candidate selection. A total of 1210 mammography images were used, from the CBIS-DDSM database. Results show that the SURF algorithm presented better performance, generating on average, for each image, 4.11 candidates considered in the internal area of the lesion, reducing exploratory space by $72 \%$, whereas the ORB generated on average 1.6 candidates with a reduction rate of $96.30 \%$.
\end{abstract}

\section{Introdução}

O câncer de mama tem sido um problema crescente para mulheres do mundo todo. De acordo com a Organização Mundial de Saúde (OMS), ele é o tipo de câncer mais comum entre mulheres, com mortalidade crescente, tanto em países subdesenvolvidos como desenvolvidos, se tornando um dos tipos mais fatais de câncer [WHO et al. 2014].

No Brasil, ele é a principal causa de morte por câncer entre as mulheres, sendo estimado 59.700 novos casos de câncer para cada ano do biênio 2018-2019, com uma incidência de cerca de 56,33 casos a cada 100 mil mulheres [INCA 2018]. Nos Estados Unidos, o National Cancer Institute (NCI) estima que 12,4\% das mulheres irão desenvolver câncer de mama durante a sua vida.

Vários métodos de prevenção têm sido desenvolvidos, mas um dos métodos mais eficazes para a detecção da lesão é a mamografia digital [Tortora and Derrickson 2016], importante para a detecção precoce do câncer, tornando o tratamento mais eficaz. No entanto, a interpretação da mamografia pode ser uma tarefa difícil até mesmo para um especialista, uma vez que a análise é afetada por diversos fatores, tais como a qualidade da imagem, experiência do radiologista e tipo de lesão. É estimado que $12 \%$ a $30 \%$ dos casos de câncer de mama deixam de ser detectados devido à má interpretação mamográfica [Evans et al. 2013]. 
Atualmente, para a correta interpretação dos exames mamográficos, são utilizadas técnicas de visão computacional e processamento de imagens, que torna o diagnóstico menos suscetível a erros por meio da identificação precisa de anomalias [Cordeiro et al. 2017][Leal et al. 2017]. Uma das técnicas utilizadas é a aplicação de algoritmos de detecção de pontos de interesse, para identificação de regiões de interesse em diferentes áreas de uma imagem. Entretanto, o uso desses detectores são aplicados em imagens mamográficas apenas para extração de características e classificação [Abbas 2016].

Desta forma, o presente trabalho propõe um novo método para seleção de candidatos à lesão em imagens mamográficas, baseado na utilização de algoritmos de detecção de pontos de interesse para geração dos candidatos. Ainda, para comprovar a eficiência da abordagem, foi analisado o comportamento dos algoritmos SURF, SIFT, BRISK e ORB, além do algoritmo Selective Search.

\section{Trabalhos Relacionados}

Algoritmos de detecção de pontos de interesse, tais como SIFT, SURF, BRISK, ORB, entre outros, têm obtido bom desempenho na identificação de objetos em imagens, como lesões em imagens mamográficas [Bianco et al. 2015]. Esses algoritmos utilizam técnicas de extração local de características baseadas nos pontos de interesses gerados, que podem ser utilizadas como dados de entrada para classificadores.

Há na literatura várias pesquisas que mostram a utilização desses algoritmos. Deshmukh e Bhosle [Deshmukh and Bhosle 2017] relatam sobre a aplicação do SURF para obtenção de vetores de características e usa diferentes classificadores em imagens mamográficas. Em conjunto com o SURF, os autores utilizaram o algoritmo PreARM, para reduzir a dimensionalidade do vetor de características do SURF. Como resultado os autores revelam que o SURF supera outros métodos no que diz respeito à distinção, repetibilidade e robustez. O algoritmo é computado mais rapidamente, e teve $96,87 \%$ de precisão para classificação das lesões em benignas ou malignas para a base DDSM.

A pesquisa de Karami et al. [Karami et al. 2017] faz uma comparação de desempenho de três algoritmos (SIFT, SURF e ORB), além da equiparação de diferentes tipos de alterações, como por exemplo a escala, a rotação e o ruído. Foram aplicados diferentes tipos de transformações na imagem e posteriormente calculado a quantidade de pontos de interesse, a taxa de correspondência e o tempo de execução necessário para cada algoritmo. Em relação ao tempo de execução, o ORB foi considerado o mais rápido, porém o SIFT obtém melhores resultados na maioria dos cenários. Quanto às imagens com ruídos o ORB e o SIFT mostram resultados semelhantes.

Na pesquisa de Salazar-Licea et al. [Salazar-Licea et al. 2014] é apresentada uma ferramenta que pode melhorar a busca de áreas suspeitas que contenham anormalidades em mamografias. Foram utilizados três métodos, entre eles o algoritmo SIFT para a localização de regiões de interesse na imagem. Os resultados sugerem que a metodologia proposta, com a utilização do SIFT, tem potencial como ferramenta para encontrar regiões suspeitas em mamografias. Entretanto, os autores pontuam que, para que o SIFT funcione da melhor maneira possível, é necessário melhorar a qualidade da imagem. 


\section{Método Proposto}

Muitos métodos de classificação no estado da arte se baseiam em técnica de janela deslizante, que gera grande quantidade de candidatos, o que requer alto custo computacional para alguns sistemas. O trabalho desenvolvido propõe um novo método para seleção de candidatos à lesão, baseado na utilização de algoritmos de detecção de pontos de interesse para geração dos candidatos. Esses candidatos são posteriormente filtrados, permitindo a redução do espaço exploratório. O fluxograma da abordagem proposta pode ser visto na Figura 1.

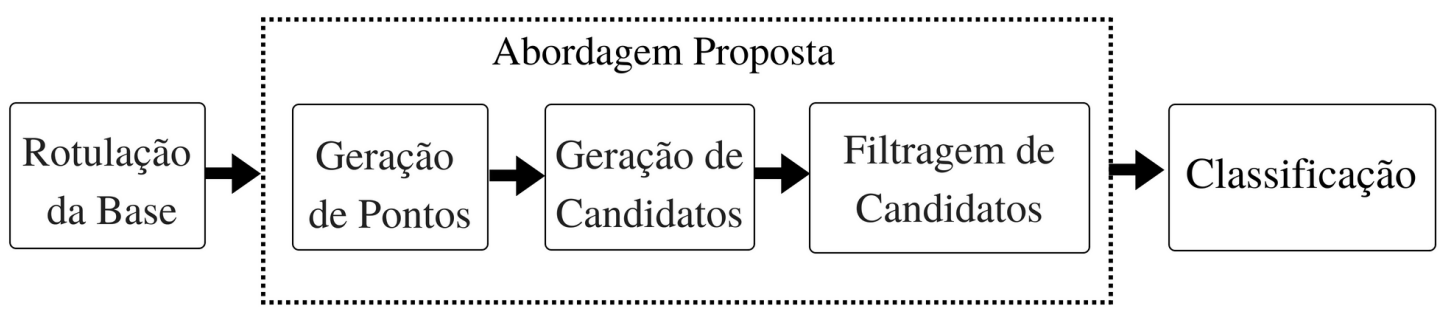

Figura 1. Fluxograma do Método Proposto

Conforme mostrado na Figura 1, inicialmente foi feita uma rotulação na base para validação dos métodos estudados. Porém, para o funcionamento do algoritmo não é necessário nenhuma rotulação. A abordagem proposta inicializa com o processo de geração de pontos de interesse, os quais servirão como base para a geração de candidatos. Como esse processo gera um número alto de candidatos, ele é seguido de uma etapa de filtragem, onde esse número será reduzido.

A abordagem proposta servirá como entrada para algoritmos de classificação, assim como representado no fluxograma acima. Nas abordagens mais recentes, como Deep Learning [Dhungel et al. 2017], o custo computacional é bastante reduzido com a diminuição no número de candidatos fornecidos como entrada aos algoritmos. Cada uma das etapas da abordagem proposta são descritas com mais detalhes nas seções a seguir.

\subsection{Rotulação da Base}

Com o conjunto de imagens ouro fornecidas pela base, onde cada imagem representa uma máscara binarizada da imagem original, no qual a cor preta representa o fundo e a cor branca representa a lesão, foi gerada a identificação das janelas contendo as lesões, para posterior análise comparativa com as janelas encontradas pelo método proposto.

Dois passos sãos utilizados para identificação da lesão na imagem ouro: o primeiro encontra contornos de objetos que estejam presentes na imagem, neste caso específico as lesões simbolizadas na cor branca e, posteriormente, um retângulo é representado englobando todos esses contornos. Esse processo pode ser observado na Figura 2.

A Figura 2 (a) mostra a imagem original, a Figura 2 (b) mostra como a imagem ouro é representada na base, a Figura 2 (c) como é identificado um retângulo englobando a lesão e a Figura 2 (d) mostra como pode ser representada a imagem original a partir das informações colhidas nos passos anteriores.

\subsection{Geração de Pontos}

Ao término da execução de um algoritmo de detecção de pontos de interesse, um conjunto de pontos distribuídos é gerado ao longo da imagem, onde cada ponto contém suas 


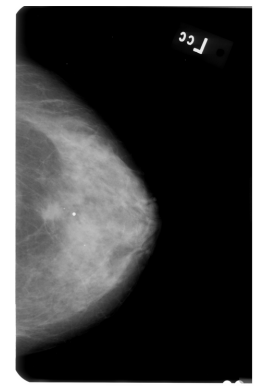

(a)

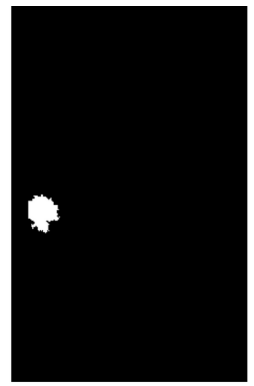

(b)

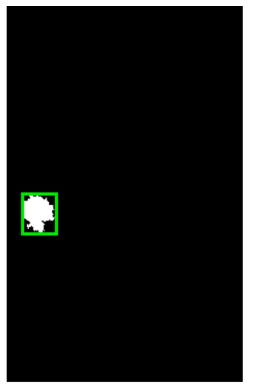

(c)

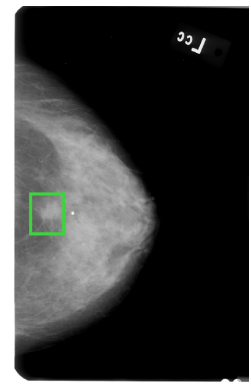

(d)

Figura 2. Rotulação da base: (a) Imagem original (b) Imagem ouro (c) Imagem de ouro com marcação (d) Imagem original com marcação

características locais relacionadas. Cada ponto pertencente ao conjunto de pontos gerados pelos algoritmos possui coordenadas $x$ e $y$, utilizadas para localizar o ponto na imagem, além de um valor referente a um diâmetro, que pode variar de ponto para ponto. A Figura 3 mostra resultados da geração de pontos de interesse em uma imagem de mamografia.

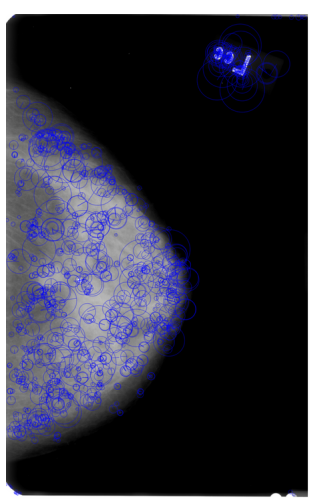

(a)

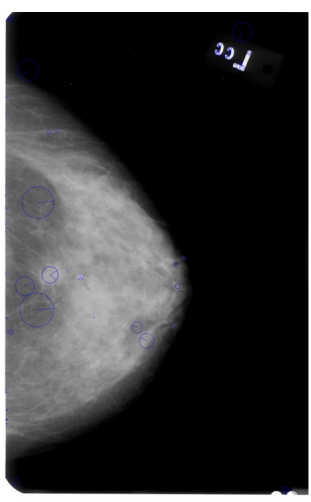

(b)

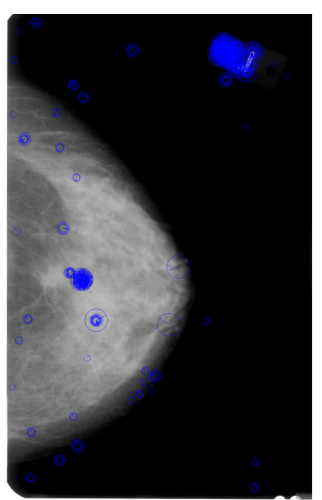

(c)

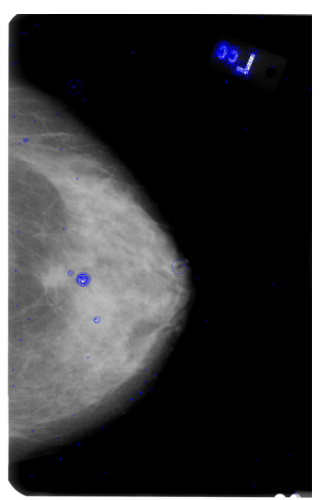

(d)

Figura 3. Geração de pontos: (a) Imagem com aplicação do SURF (b) Imagem com aplicação do SIFT (c) Imagem com aplicação do ORB (d) Imagem com aplicação do BRISK

Para os exemplos apresentados acima, é possível observar que o algoritmo SURF, na Figura 3 (a), gerou grande quantidade de pontos distribuídos ao longo da área que representa a mama na imagem, além da área onde o rótulo está presente. Para o SIFT, na Figura 3 (b), essa quantidade foi menor e, mesmo assim, foi gerado pontos na área do rótulo. Para o algoritmo ORB, na Figura 3 (c), a quantidade de pontos gerados foi maior que o SIFT porém a distribuição foi um pouco menor e muitos pontos ficaram sobrepostos aos outros, além do grande número de pontos gerados no rótulo da imagem. Por fim, na Figura 3 (d), o algoritmo BRISK mostra uma quantidade de geração de pontos menor que o ORB, porém com distribuição parecida.

Originalmente, a distribuição dos pontos na imagem não segue um padrão bem definido e, desta forma, dependendo do problema a ser abordado, uma grande quantidade de pontos podem ser removidos sem afetar o resultado final desejado. Quais pontos devem ser levados em consideração ou podem ser descartados, será explicado com mais detalhes na seção 3.4 . 


\subsection{Geração de Candidatos}

Um candidato é gerado a partir da região delimitada pelo valor referente ao diâmetro fornecido pelos algoritmos para cada ponto de interesse. Sendo assim, podemos representar essa área como uma janela na imagem original. Uma janela é definida pelo quadrado mínimo que engloba a circunferência relacionada a cada ponto. Os algoritmos são aplicados sem a realização prévia de nenhuma técnica de pré-processamento na imagem original. Nesta etapa, todas as janelas geradas são consideradas no conjunto de candidatos, que posteriormente serão filtradas. Podemos observar na Figura 4 como é realizado o processo de geração de candidatos.

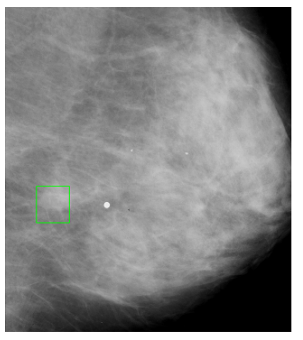

(a)

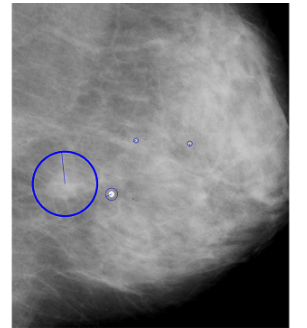

(b)

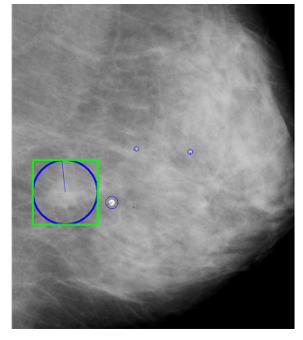

(c)

Figura 4. Seleção de Candidatos: (a) Localização da lesão na imagem original (b) Imagem com aplicação do SURF (c) Imagem com aplicação do SURF e seleção de uma janela referente a um ponto de interesse.

Com base em informações obtidas na etapa de Rotulação da Base, como mostrado na seção 3.1, na Figura 4 (a) podemos observar a localização da lesão na imagem original. Na Figura 4 (b) é possível observar o resultado da execução do algoritmo SURF, onde um ponto de interesse é gerado na imagem original. Na Figura 4 (c) pode-se observar como um candidato é gerado a partir da janela representativa da área relacionada ao ponto de interesse. Para o exemplo específico apresentado, o candidato gerado pode ser utilizado como entrada para um classificador e obter um bom resultado na detecção da lesão na imagem original.

\subsection{Filtragem de Candidatos}

Uma imagem de mamografia digital possui normalmente, além do conteúdo relacionado a imagem radiografada da mama e, dependendo do posicionamento da captura, informações referentes ao músculo peitoral maior, rótulos e ruídos que não devem ser levados em consideração, uma vez que não refletem em nenhum momento a lesão presente na mama. Como mostrado da seção 3.3 a área relacionada a cada ponto de interesse possui uma janela candidata associada. Os algoritmos podem gerar grande quantidade de janelas com áreas muito extensas ou muito pequenas, onde em nenhum dos casos, irá refletir um tamanho normal da lesão. Desta maneira, para remover janelas presentes em locais irrelevantes na imagem e janelas com tamanhos desproporcionais ao tamanho da lesão, são aplicados os filtros descritos a seguir.

\subsubsection{Filtro de tamanho}

Os valores referentes ao menor e maior tamanho de área que uma lesão pode possuir foram armazenados na epata de Rotulação da Base e utilizados na aplicação de um filtro 
onde os candidatos que possuíram uma área menor que a menor área possível de ser uma lesão foram descartados. Seguindo a mesma lógica, os candidatos que possuíram áreas maiores que a maior área possível a ser uma lesão também foram descartados. Os resultados da redução do espaço de exploração dos candidatos após a aplicação deste filtro são apresentados com detalhes na seção 5.

\subsubsection{Filtro de Fundo}

Um filtro foi aplicado para desconsiderar os candidatos que estão presentes em áreas da imagem consideradas irrelevantes, tais como áreas que estão relacionados aos rótulos e ruídos presentes na imagem, ou que englobam uma parte considerável do fundo da imagem. Essa etapa possui dois passos que são apresentados a seguir.

Primeiro passo: cada janela pertencente ao conjunto de candidatos formado na etapa de Seleção de candidatos é inicialmente binarizada, utilizando a técnica de limiarização de OTSU [Otsu 1979]. Ao término deste passo, os pixels pertencentes a cada janela candidata passou a ser considerado como branco ou preto.

Segundo passo: após uma janela candidata ser binarizada é possível percorrer e analisar sua matriz representativa com o objetivo de inferir se essa janela deve ser descartada ou não do conjunto final. Ao percorrer a matriz, caso algum elemento possua o valor 0 , significa que esse pixel tem a cor preta, ou seja, esse pixel representa parte do fundo da imagem. Em contrapartida, caso o elemento possua o valor 255 significa que esse pixel não representa parte do fundo da imagem.

Ao término da verificação das matrizes das janelas candidatas separadamente, foi possível medir a relação de pertinência entre cada janela e o fundo da imagem original. Uma vez que a região da mama está nas áreas mais claras da imagem, o filtro de fundo considerou como candidato final, após o processo de binarização, apenas aqueles que possuíam a maior parte da sua vizinhança da cor branca. Com isso, os candidatos com vizinhança com pixels pretos, tais como rótulos, ruído ou fundo, são eliminados.

A Figura 5 mostra passo a passo como o processo de aplicação do filtro de fundo é realizado. Primeiramente, é possível observar a imagem original contendo apenas uma janela candidata identificada. Esta janela selecionada se transforma então em uma subimagem candidata. Posteriormente, essa subimagem candidata é binarizada e, por fim, serviu de entrada para o algoritmo "Verificador de fundo".

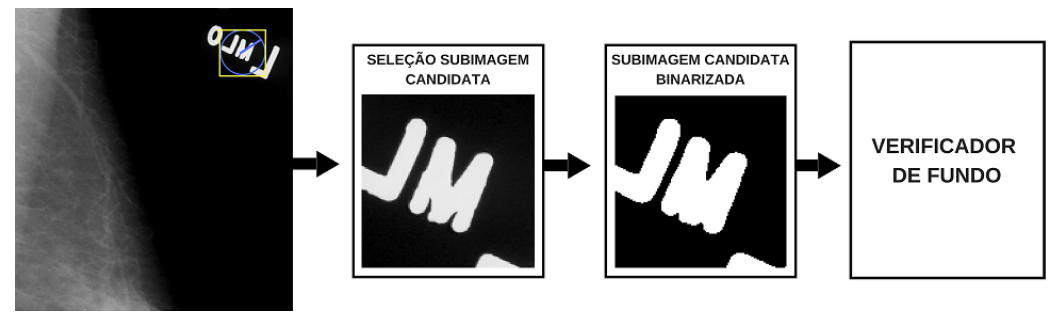

Figura 5. Fluxo de execução do filtro de fundo

Por fim, após a etapa de filtragem é formado o conjunto final de candidatos à lesão na imagem mamográfica. A Figura 6 mostra o resultado final da etapa de filtragem de 
candidatos. A Figura 6 (a) mostra a imagem original, a Figura 6 (b) mostra a imagem com aplicação do algoritmo SURF e por fim, a Figura 6 (c) mostra a imagem após a etapa de filtragem.

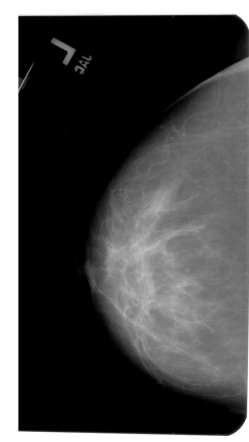

(a)

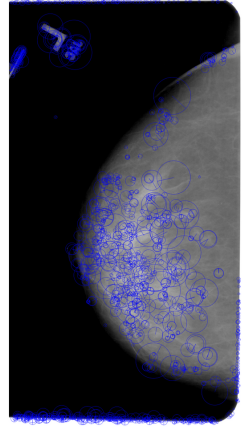

(b)

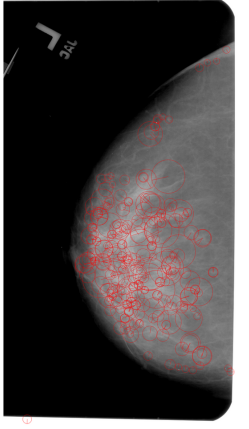

(c)

Figura 6. Pós-Processamento: (a) Imagem original (b) Imagem com aplicação do algoritmo SURF (c) Imagem após a etapa de pós-processamento.

\section{Metodologia}

\subsection{Ambiente Experimental}

A base utilizada foi a CBIS-DDSM (Curated Breast Imaging Subset of DDSM) que contém 6775 casos de estudo relacionados à 6671 pacientes, totalizando 10239 imagens, além de imagens digitais de mamografia capturadas de ângulos diferentes. Em resumo, a CBIS-DDSM é uma versão atualizada e padronizada da base DDSM. Para o presente trabalho foram utilizadas 1210 imagens.

Os algoritmos implementados no presente trabalho foram desenvolvidos a partir da linguagem de programação Python com auxílio da biblioteca OpenCV em um computador com processador i7 e 8GB de memoria RAM.

\subsection{Algoritmos Implementados}

O Scale Invariant Feature Transform (SIFT) é um algoritmo que permite a detecção e extração local de características de uma imagem. Apesar de ser eficiente, requer um grande custo computacional. Desta forma, surgiram diversas variantes e extensões melhoradas do SIFT [Karami et al. 2017]. Uma dessas variantes é o Speeded-Up Robust Features (SURF), que funciona mais rápido que o SIFT sem reduzir a qualidade dos pontos detectados. Baseado na matriz Hessiana, o SURF inclui etapas de detecção e localização do ponto de interesse usando uma matriz [Rodrigues et al. 2014].

O Oriented FAST and Rotated BRIEF (ORB) é outra alternativa para o SIFT e SURF. Baseado no FAST e no BRIEF, técnicas que possuem bom desempenho e baixo custo, o ORB aborda algumas limitações das técnicas citadas, principalmente a falta de invariância rotacional do BRIEF, e adiciona um componente de orientação rápido e preciso ao FAST [Rublee et al. 2011]. Assim como o ORB, o Binary Robust Invariant Scalable Keypoints (BRISK) melhora os conceitos do BRIEF. Considerado um descritor binário, é um método para deteç̧ão, descrição e correspondência de pontos de interesse. Além disso, tem um desempenho adaptável e de alta qualidade, com um custo computacional menor que o SURF [Leutenegger et al. 2011]. 
Técnicas baseadas em janela deslizante são utilizadas para localização de objetos em imagens. Nessa abordagem, um tamanho é definido para a janela e um valor referente ao deslocamento horizontal e vertical ao longo da imagem. No entanto, o espaço de busca é muitas vezes muito grande, tornando a busca exaustiva um custo computacional elevado [Uijlings et al. 2013].

Além da aplicação dos algoritmos descritos acima, foi implementada a técnica $S e$ lective Search, que é baseada em uma abordagem chamada Region Proposal, uma solução para melhorar os problemas da busca exaustiva com janela deslizante e bastante utilizada para geração de candidatos na área de reconhecimento de objetos. A técnica Selective Search passa pelo mesmo processo de filtragem que os demais algoritmos foram submetidos para formar o conjunto final de candidatos, removendo janelas relacionadas ao fundo, rótulos e ruídos. A Figura 7 apresenta a aplicação da etapa de filtragem de candidatos onde o algoritmo Selective Search foi aplicado.

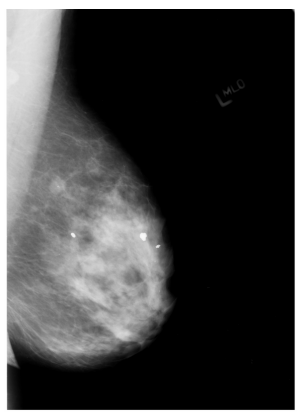

(a)

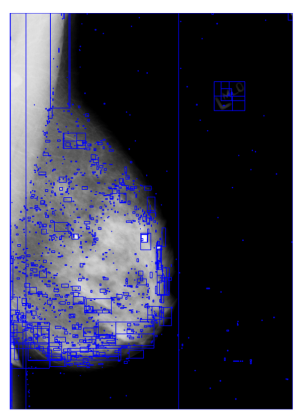

(b)

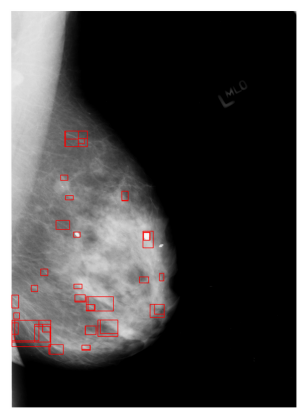

(c)

Figura 7. Filtragem de Candidatos no Selective Search: (a) Imagem original (b) Imagem com aplicação Selective Search (c) Imagem após etapa de filtragem no Selective Search.

\subsection{Verificação de Acurácia da localização de Candidatos}

A partir dos candidatos finais gerados após a etapa de filtragem, é possível medir a precisão de cada algoritmo na localização da lesão. Para isso, foi verificado a localização de cada candidato na imagem original para ser comparado com a localização real da lesão. Desta forma, é possível identificar se esse candidato está dentro, sobreposto ou próximo da área da lesão. É possível observar na Figura 8 como essa averiguação foi realizada. O quadrado em verde mostrado na Figura 8 representa o posicionamento da janela representando a localização real da lesão na imagem original. O quadrado em amarelo representa a localização de um candidato.

Conforme mostrado na Figura 8, para que um candidato seja considerado dentro da área da lesão, o tamanho da sua área deve ser menor que a área da janela da lesão e, todo seu conteúdo deve estar presente no conteúdo da janela da lesão, assim como representado na Figura 8 (a). Em caso de ser considerado sobreposto à lesão, o candidato deve ter necessariamente o tamanho de área maior que o tamanho da janela da lesão e, todo conteúdo da janela da lesão deve estar presente no conteúdo do candidato, como na Figura 8 (b). Por fim, para um candidato ser considerado próximo da lesão, a única exigência é que, o conteúdo da janela do candidato tenha uma relação de intersecção ao conteúdo da janela da lesão, como na 8 (c). 


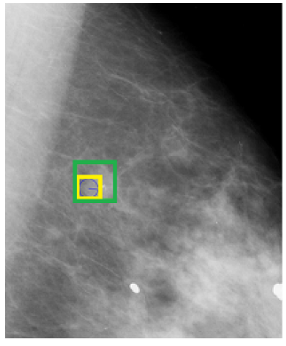

(a)

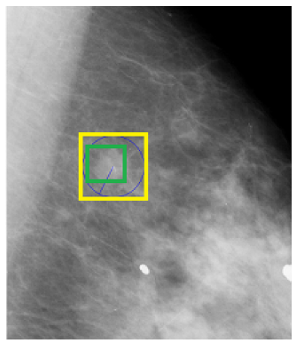

(b)

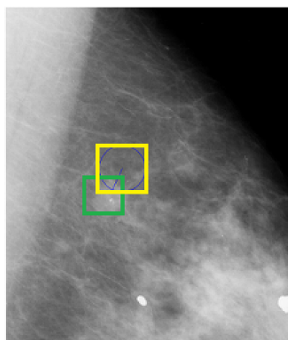

(c)

Figura 8. (a) Candidato dentro da área da lesão (b) Candidato sobreposto da área da lesão (c) Candidato próximo da área da lesão

\subsection{Métricas}

Intersecção pela União (IoU) é uma métrica de avaliação usada para medir a precisão de um detector de objetos em um conjunto de dados específico. [Rosebrock 2016]. O valor do IoU é dado pelo resultado da divisão entre a intersecção sobre a união entre duas janelas. Este valor quanto mais próximo de 1 significa que uma janela está mais alinhada sobre a outra, em termos de sobreposição. É possível observar como é representado o cálculo do $I o U$ na Equação 1, onde $I$ corresponde ao valor referente a intersecção e $U$ corresponde ao valor da união.

$$
I o U=\frac{I}{U}
$$

Para que seja possível calcular o IoU, precisamos ter informações referentes a duas janelas: a primeira para representar a localização real previamente fornecida do objeto na imagem, e a segunda para representar a localização do objeto que desejamos detectar.

\section{Resultados Obtidos}

Assim como mostrado na seção 3.4, para todos os algoritmos foi aplicado a etapa de filtragem para selecionar o conjunto final de candidatos. Além disso, utilizando o processo realizado e apresentado na seção 4.3, foi aplicado a métrica IoU para cada candidato. Para configuração dos algoritmos analisados, foram utilizados os parâmetros definidos por padrão.

Inicialmente foi realizada uma análise da quantidade de janelas obtidas por cada algoritmo e o impacto do processo de filtragem no número de candidatos resultantes. A Tabela 1 apresenta na primeira coluna os nomes dos algoritmos executados, a segunda coluna apresenta a quantidade média de candidatos que cada algoritmo gerou sem a filtragem, a terceira coluna apresenta essa quantidade após a filtragem e, por último, a quarta coluna mostra a redução atingida em porcentagem do espaço exploratório.

Foi observado que o algoritmo SURF foi o que obteve menor redução do espaço exploratório, o que não representa um resultado ruim, uma vez que a quantidade de candidatos restantes é maior em comparação aos outros algoritmos, podendo aumentar a chance de localizar a lesão mesmo sem ser necessário explorar áreas irrelevantes na imagem.

Também foi feita uma análise comparando o número de janelas obtidas pelos algoritmos com o método da janela deslizante. Sabendo que o tamanho médio das imagens 
Tabela 1. Resultado da etapa de filtragem de candidatos: Redução do espaço exploratório

\begin{tabular}{llllll}
\hline Algoritmo & \multicolumn{2}{l}{ Sem Filtragem } & \multicolumn{2}{l}{ Com Filtragem } & Redução \\
\hline SURF & 1176,21 & $\pm 647,82$ & 329,83 & $\pm 195,28$ & $72 \%$ \\
SIFT & 102,73 & $\pm 77,25$ & 8,8 & $\pm 7,56$ & $91,50 \%$ \\
BRISK & 174,33 & $\pm 144,74$ & 3,19 & $\pm 8,50$ & $98,20 \%$ \\
ORB & 334,98 & $\pm 138,65$ & 32,49 & $\pm 36,73$ & $96,30 \%$ \\
SS & 862,7 & $\pm 396,80$ & 17,21 & $\pm 13,58$ & $98,10 \%$ \\
\hline
\end{tabular}

é de $2652 \times 1569$ pixels, e aplicando uma janela deslizante de tamanho $200 \times 200$ pixels com deslocamento horizontal e vertical de 5 pixels a cada interação, para percorrer toda a imagem se faz necessário a geração de 135.036 janelas candidatas em média. Após a aplicação dos algoritmos, foi obtida uma redução relevante no espaço exploratório. A Tabela 2 apresenta esses resultados, onde na primeira coluna mostra os nomes dos algoritmos analisados, a segunda e quarta colunas mostram informações sobre a quantidade média de candidatos gerados por cada algoritmo, com e sem a etapa de filtragem, respectivamente e, a terceira e quinta colunas mostram as reduções obtidas.

\begin{tabular}{|c|c|c|c|c|}
\hline \multirow{2}{*}{ Algoritmos } & \multicolumn{2}{|c|}{ Sem Filtragem } & \multicolumn{2}{|c|}{ Com Filtragem } \\
\hline & \# Candidatos & Redução & \# Candidatos & Redução \\
\hline SURF & 1176,21 & $99,13 \%$ & 329,83 & $99,76 \%$ \\
\hline SIFT & 102,73 & $99,92 \%$ & 8,8 & $99,98 \%$ \\
\hline BRISK & 174,33 & $99,87 \%$ & 3,19 & $99,98 \%$ \\
\hline ORB & 334,98 & $99,75 \%$ & 32,49 & $99,97 \%$ \\
\hline $\mathrm{SS}$ & 862,7 & $99,36 \%$ & 17,21 & $99,98 \%$ \\
\hline
\end{tabular}

A redução do espaço exploratório após a utilização de cada algoritmo chegou perto de $100 \%$ para todos os casos, mostrando que a técnica baseada em janela deslizante, além de necessitar de um alto custo computacional, tende a gerar muitos candidatos desnecessários para o problema.

Assim como visto na seção 4.3 e mostrado na Figura 8, um candidato à região suspeita pode estar dentro, sobreposto ou próximo a localização real da lesão na imagem original. A Tabela 3 apresenta o resultado da análise desses candidatos onde na primeira coluna mostra o nome do algoritmo, a segunda coluna mostra a quantidade média de candidatos, a terceira coluna mostra a quantidade média de candidatos considerados dentro da lesão, a quarta coluna mostra os candidatos considerados sobrepostos, a quinta coluna apresenta os candidatos considerados próximos à lesão e, por último, é mostrado a quantidade média de candidatos considerados como falso positivos.

Conforme apresentado na Tabela 3, foi observado que o algoritmo SURF apresentou maior precisão na geração de candidatos, pois obteve um maior número de janelas dentro, sobreposta ou próxima à lesão da imagem. Comparado aos outros algoritmos, o SURF apresenta em média 4,11 janelas dentro da região da lesão, enquanto que os outros algoritmos nem sempre obtém um candidato na região da lesão, obtendo médias abaixo de 
Tabela 3. Resultado da aplicação dos algoritmos na seleção dos candidatos à regiões suspeitas

\begin{tabular}{|c|c|c|c|c|c|}
\hline \multirow{2}{*}{ Algoritmo } & \multirow{2}{*}{ \# Candidatos } & \multicolumn{3}{|c|}{ Verdadeiro Positivos } & \multirow{2}{*}{ Falso Negativos } \\
\hline & & \# Dentro & \# Sobreposto & \# Próximo & \\
\hline SURF & 329,83 & 4,11 & 0,3 & 16,5 & 308,88 \\
\hline SIFT & 8,8 & 0,62 & 0,05 & 0,55 & 7,58 \\
\hline BRISK & 3,19 & 0,08 & 0 & 0,16 & 2,96 \\
\hline ORB & 32,49 & 0,7 & 0 & 1,6 & 30,19 \\
\hline SS & 17,21 & 0,38 & 0 & 0,37 & 16,45 \\
\hline
\end{tabular}

1. O mesmo se aplica para janelas próximas à região de interesse. O algoritmo ORB apresentou um bom resultado ao gerar em média 1,6 candidatos próximos, porém apresentou valores baixos em relação aos outros grupos.

\section{Considerações Finais e Trabalhos Futuros}

O trabalho desenvolvido propôs um novo método para seleção de candidatos à região suspeita de lesão em imagens mamográficas, baseado na utilização de algoritmos de detecção de pontos de interesse para geração dos candidatos. Para validação da proposta foram analisados os algoritmos SURF, SIFT, BRISK e ORB, além do algoritmo Selective Search para 1210 imagens de mamografia da base CBIS-DDSM.

A redução do espaço exploratório e o resultado da geração de candidatos dentro da lesão pelo algoritmo SURF, podem servir de dados de entrada a um classificador, beneficiando a detecção de lesão em imagens de mamografia. Em trabalhos futuros, realizaremos análises adicionais aplicando etapas de pré-processamento para verificar a eficiência desses algoritmos levando em consideração contraste e também remoção de outras áreas que não há possibilidades de conter uma lesão.

\section{Agradecimentos}

Agradecemos a Fundação de Amparo a Ciência e Tecnologia de Pernambuco (FACEPE) e ao Conselho Nacional de Desenvolvimento Científico e Tecnológico (CNPq) pelo apoio intelectual fornecido e o subsídio necessário para o desenvolvimento da pesquisa.

\section{Referências}

Abbas, Q. (2016). Deepcad: A computer-aided diagnosis system for mammographic masses using deep invariant features. Computers, 5(4):28.

Bianco, S., Mazzini, D., Pau, D., and Schettini, R. (2015). Local detectors and compact descriptors for visual search: a quantitative comparison. Digital Signal Processing, 44:1-13.

Cordeiro, F. R., Santos, W. P. d., and Silva-Filho, A. G. (2017). Analysis of supervised and semi-supervised growcut applied to segmentation of masses in mammography images. Computer Methods in Biomechanics and Biomedical Engineering: Imaging \& Visualization, 5(4):297-315.

Deshmukh, J. and Bhosle, U. (2017). Surf features based classifiers for mammogram classification. In Wireless Communications, Signal Processing and Networking (WiSPNET), 2017 International Conference on, pages 134-139. IEEE. 
Dhungel, N., Carneiro, G., and Bradley, A. P. (2017). A deep learning approach for the analysis of masses in mammograms with minimal user intervention. Medical image analysis, 37:114-128.

Evans, K. K., Birdwell, R. L., and Wolfe, J. M. (2013). If you don't find it often, you often don't find it: Why some cancers are missed in breast cancer screening. PloS one, 8(5):e64366.

INCA (2018). Estimativa 2018 - incidência de câncer no brasil.

Karami, E., Prasad, S., and Shehata, M. (2017). Image matching using sift, surf, brief and orb: Performance comparison for distorted images. arXiv preprint arXiv:1710.02726.

Leal, I., Aires, K., and Veras, R. (2017). Diagnóstico de benignidade e malignidade em imagens mamográficas utilizando aprendizado profundo para extração das regiões de interesse e Índices de diversidade filogenéticas para classificação. Anais do XIV Encontro Nacional de Inteligência Artificial e Computacional, pages 250-261.

Leutenegger, S., Chli, M., and Siegwart, R. Y. (2011). Brisk: Binary robust invariant scalable keypoints. In Computer Vision (ICCV), 2011 IEEE International Conference on, pages 2548-2555. IEEE.

Otsu, N. (1979). A threshold selection method from gray-level histograms. IEEE transactions on systems, man, and cybernetics, 9(1):62-66.

Rodrigues, L. A., Silva, F. A. d., Pereira, D. R., Artero, A. O., and Piteri, M. A. (2014). Detecção e reconhecimento de objetos em imagens utilizando algoritmos de extração de pontos chave. In Colloquium Exactarum. ISSN: 2178-8332, volume 6, pages 86103.

Rosebrock, A. (2016). Intersection over union (iou) for object detection. Online] http://www. pyimagesearch. com/2016/11/07/intersection-overunion-iou-for-objectdetection.

Rublee, E., Rabaud, V., Konolige, K., and Bradski, G. (2011). Orb: An efficient alternative to sift or surf. In Computer Vision (ICCV), 2011 IEEE international conference on, pages 2564-2571. IEEE.

Salazar-Licea, L. A., Mendoza, C., Aceves, M., Pedraza, J., and Pastrana-Palma, A. (2014). Automatic segmentation of mammograms using a scale-invariant feature transform and k-means clustering algorithm. In Electrical Engineering, Computing Science and Automatic Control (CCE), 2014 11th International Conference on, pages 1-6. IEEE.

Tortora, G. J. and Derrickson, B. (2016). Corpo Humano-: Fundamentos de Anatomia e Fisiologia. Artmed Editora.

Uijlings, J. R., Van De Sande, K. E., Gevers, T., and Smeulders, A. W. (2013). Selective search for object recognition. International journal of computer vision, 104(2):154171.

WHO et al. (2014). WHO position paper on mammography screening. World Health Organization. 